Research in Astron. Astrophys. Vol.0 (20xx) No.0, 000-000

http://www.raa-journal.org http://www.iop.org/journals/raa

$\boldsymbol{R}$ esearch in

(LTEX: RAA-2018-0097.R2.tex; printed on April 19, 2022;

Astronomy and

$11: 52)$

Astrophysics

\title{
Mineralogy of northern nearside mare basalts
}

\author{
Zhenchao Wang ${ }^{1,2}$, Yunzhao $\mathrm{Wu}^{3,2 *}$, Xiaomeng Zhang ${ }^{4}$ and $\mathrm{Yu} \mathrm{Lu}^{5,6}$ \\ ${ }^{1}$ Key Laboratory of Surficial Geochemistry, Ministry of Education, Department of Earth Sciences, \\ Nanjing University, Nanjing 210023, China; \\ 2 Space Science Institute, Macau University of Science and Technology, Macau, China; \\ 3 Key Laboratory of Planetary Sciences, Purple Mountain Observatory, Chinese Academy of Sciences, \\ Nanjing 210034, China; \\ 4 Xuzhou Bureau of Land and Resources, Xuzhou 221000, China; \\ ${ }^{5}$ School of Geographic and Oceanographic Sciences, Nanjing University, Nanjing, 210023, China; \\ 6 Jiangsu Center for Collaborative Innovation in Geographical Information Resource Development \\ and Application, Nanjing, 210023, China;
}

Received 20xx month day; accepted 20xx month day

\begin{abstract}
The mineralogy of mare basalts reflects the chemical composition of the magma source, as well as the physical and chemical environment of the rock's formation. It is significant for understanding the thermal evolution of the Moon. In this study, the spatial distribution of the mineralogy of the lunar northern nearside basalts was mapped using the Moon Mineralogy Mapper $\left(\mathrm{M}^{3}\right)$ data. The study area, which is an elongated mare, Mare Frigoris and northern Mare Imbrium, was mapped and characterized into 27 units based on multi-source data, including spectrum, terrain, and element abundance. We extracted $177 \mathrm{M}^{3}$ spectra from fresh craters. Spectral parameters such as absorption center and band area ratio (BAR) were obtained through data processing. The variation of mafic mineral of this region was acquired by analyzing these parameters. The basaltic units in eastern Mare Frigoris, which are older, have been found to be dominated by clinopyroxene with lower $\mathrm{CaO}$ compared to the returned lunar samples; this is similar to older basaltic units in Mare Imbrium. The basaltic units of western Mare Frigoris and Sinus Roris which are younger have been found to be rich in olivine. The late-stage basalts in Oceanus Procellarum and Mare Imbrium show the same feature. These wide-spread olivine-rich basalts suggest the uniqueness in the evolution of the Moon. Geographically speaking, Mare Frigoris is an individual mare, but the magma source region have connections with surrounding maria in consideration of mineral differences between western and eastern Frigoris, as well as mineral similarities with maria at the same location.
\end{abstract}

Key words: Mare Frigoris; Moon Mineralogy Mapper; spectra; basalt unit; mineralogy

\section{INTRODUCTION}

Mare basalt covers $17 \%$ of the surface of the Moon and accounts for approximately $1 \%$ of the lunar crust (Head 1976). Its spatial distribution, composition, and volume record important information of the magmatic activity of the Moon. Research into its mineral components aids in the understanding of the magma source and characterizes the pressure, temperature, cooling rate, and other physical parameters of the magmatic state and processes. In addition, the mineralogy of mare basalts is an important scientific focus of the lunar exploration.

\footnotetext{
${ }^{*}$ Corresponding author: Yunzhao Wu (wu@pmo.ac.cn)
} 
Among the lunar maria, Mare Frigoris is very special. Most maria are circular; however, Mare Frigoris is very long and narrow (approximately $1800 \mathrm{~km}$ long with a maximum width of $200 \mathrm{~km}$ ). Although Mare Frigoris is a separate mare geographically, its basalts may be associated with the adjacent maria (such as western Mare Frigoris and Oceanus Procellarum, southern Mare Frigoris and northern Mare Imbrium). Therefore, this study chose this long and narrow mare as the major research area to analyze the variation in mineral compositions in different locations. Further, it makes a comparison with the mineral compositions of adjacent maria (northern Mare Imbrium basalts) to discuss its role in the thermal evolution of the Moon.

Fig. 1 shows the focus area of this study. It centers around Mare Frigoris, including Sinus Roris in western and northern Mare Imbrium. Mare Frigoris is located in Procellarum KREEP Terrane (PKT) on the nearside of the Moon (Jolliff et al. 2000), at $56.0^{\circ} \mathrm{N}$ central latitude and $1.4^{\circ} \mathrm{E}$ central longitude. Its west side is linked to Oceanus Procellarum by Sinus Roris. Whitford-Stark (1990) drew the geological map of Mare Frigoris based on ground observation and Lunar Orbiter data, and revealed that the oldest and the youngest basalts were located in the northeast and southwest, respectively. Hiesinger et al. (2010) divided 37 geological units using the false color images of Clementine UV/VIS, and calculated the model age using the crater size-frequency distribution (CSFD) method. The results showed that the youngest basalts were located in Sinus Roris; four young Eratosthenian basalts were located in the middle of Mare Frigoris, northern Plato crater and northwest of Baily crater; the remaining old geological units were formed in the late Mare Imbrium, aged between 3.4 and $3.8 \mathrm{Ga}$. Kramer et al. (2015) divided Mare Frigoris into 26 geological units based on Clementine, Lunar Prospector (LP), and Lunar Reconnaissance Orbiter (LRO) data. With the exception of two units in the west, where the contents of iron and titanium reached the levels of medium iron $\&$ low titanium and medium iron $\&$
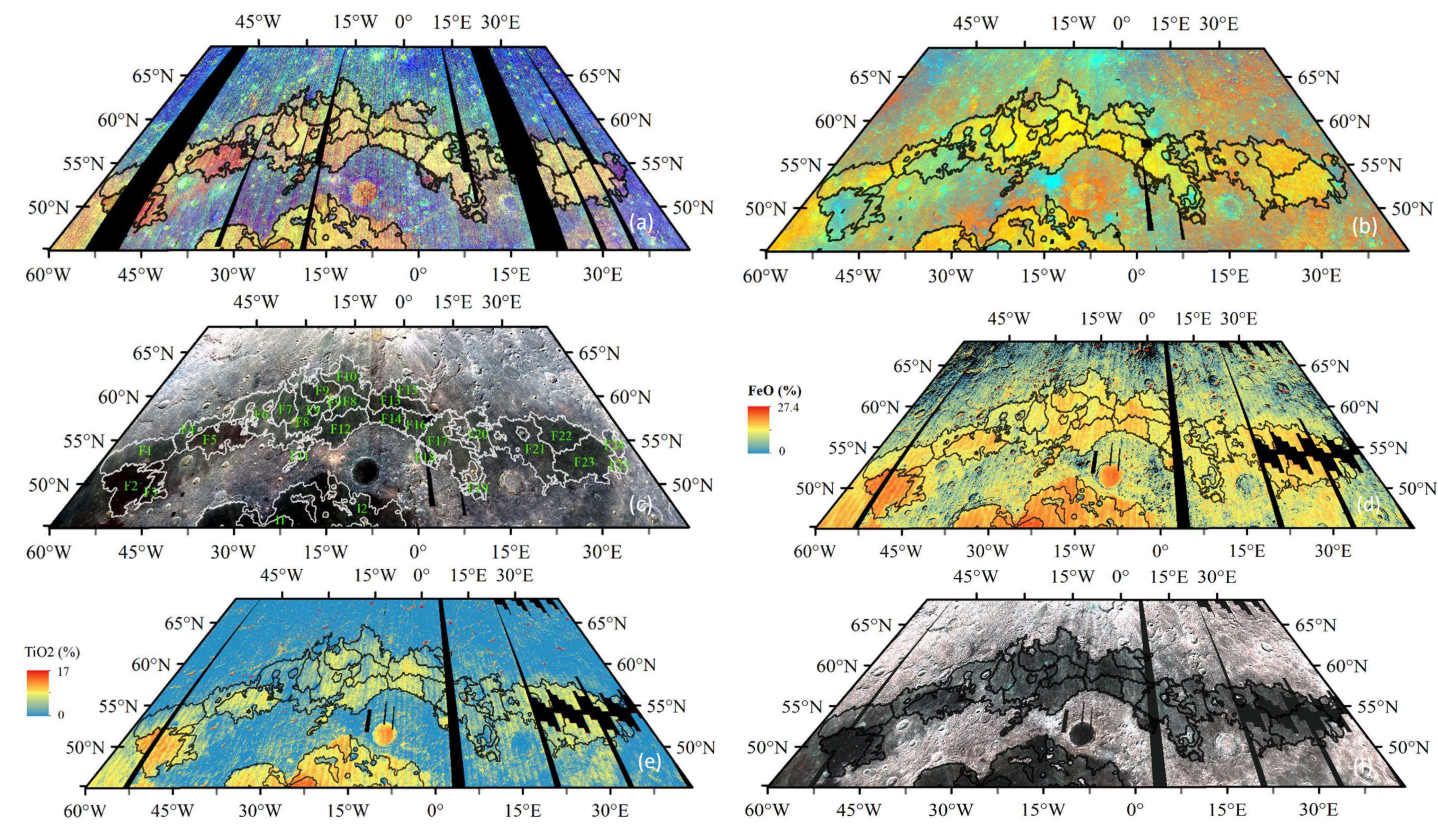

Fig. 1 Images of the study area, where black/white lines delineate the geological units divided in this study. (a) $\mathrm{M}^{3}$ integrated band depth (IBD) image (R-1000 nm IBD; G-2000 nm IBD; B-albedo of 1548 $\mathrm{nm}$ ). (b) Clementine color composite ratio image (R-750/415 nm, G-750/950 nm, B-415/750 nm). (c) Clementine false color composite image (R-950 nm; G-750 nm; B-415 nm), in which F1-F25 represents 25 geological units of Mare Frigoris, and I1 and I2 represent two geological units of Mare Imbrium. (d) The Chang'E-1 interference imaging spectroscopy (IIM) FeO content diagram. (e) $\mathrm{IIM} \mathrm{TiO}_{2}$ content diagram; (f) IIM false color composite image (R-891 nm; G-739 nm; B-618 nm). 
medium titanium, respectively, the majority of the remaining areas were characterized as high-alumina basalts, characterized by low titanium to extremely low titanium, low iron, and high alumina,

The Clementine UV/VIS data ranges from 415 to $1000 \mathrm{~nm}$ with only five bands. The spectral resolution is low, which restricts the reliable identification of the minerals. The India's Chandrayaan-1 Moon Mineralogy Mapper $\left(\mathrm{M}^{3}\right)$ data have a wider range of wavelengths and higher spectral resolution. They can make an approximate description of the spectral curve continuously, so as to more accurately infer the mineral composition. The study obtains the spectral absorption characteristics of fresh craters by using of the $\mathrm{M}^{3}$ data to identify the mineral composition of Mare Frigoris and northern Mare Imbrium. Then, it compares the composition with those of adjacent maria to discuss its regional geological significance.

\section{DATA}

The $\mathrm{M}^{3}$ Level 2 (L2) data with spatial resolution of $140 \mathrm{~m} /$ pixel were used for the mineral analysis. In addition, this study adopts a variety of other data for the division of the stratigraphic units. Of these, the standard Clementine color ratio composite (R, 750/415, G, 750/950, B, 415/750) map and the standard $\mathrm{M}^{3}$ integrated band depth (IBD) composite (R: $1000 \mathrm{~nm}$ IBD; G: the $2000 \mathrm{~nm}$ IBD; and B: reflectance at $1480 \mathrm{~nm}$ ) were used as base maps for separating basaltic units. In the two mosaic images the stitching traces between orbital boundaries are very obvious, which makes it difficult for the separation of different geologic units. The Lunar Reconnaissance Orbiter Camera (LROC) Wide Angle Camera (WAC) 7-channel composite (Wagner et al. 2015) and the Chang'E-1 interference imaging spectroscopy (IIM) data calibrated in Wu et al. (2013) eliminate the tile-shaped splicing boundary. These non-trace mosaics are conducive to the preparation of the geological map, though they are partially missing and unable to completely cover the study area. Moreover, the $\mathrm{M}^{3}$ images containing thermal emission radiation, the WAC low-Sun mosaic and 30 m LOLA DEM show morphology information hence contributed to the separation of the basaltic units (Wu et al. 2018a). The $\mathrm{FeO}$ and $\mathrm{TiO}_{2}$ maps were from the original IIM 2C level data (Wu 2012). All the data except IIM were from the Planetary Data System (PDS).

\section{METHODOLOGY}

\subsection{Separation of Stratigraphy}

The stratigraphic division, which is important for the interpretation of mineralogy and geology, is not easy work because of the space weathering, contamination by the ejecta and lack of perfectly calibrated data. Following the methodology developed in Wu et al. (2018a), the separation of geologic units of this study was derived using diverse datasets including Clementine band ratio composite, $\mathrm{FeO}$ abundances, IIM color composite, LROC WAC mosaic composite, $\mathrm{M}^{3}$ IBD and $\mathrm{M}^{3}$ enhanced color, LROC WAC low-Sun image). All of these images were input in ArcGis and stretched for contrast enhancement.

\subsection{Collection of Spectra}

To avoid space weathering, the spectra from small fresh craters with diameters of $<\sim 1 \mathrm{~km}$ or slightly larger, and the mean spectra calculated from several pixels of the individual crater have been often used in previous publications (Kramer et al. 2008; Staid \& Pieters 2001; Whitten et al. 2011; Kaur et al. 2013). The extracted spectra from craters with diameters of $\sim 1 \mathrm{~km}$ easily contain the compositions of the underlying stratigraphy because these craters of this size could excavate depths of approximately 84 $\mathrm{m}$ (Melosh 1989). If a crater has a lower resolution than that of $\mathbf{M}^{3}$, the spectra are not from fresh crater itself; instead, they contain shadows, melts, and soils. Through trial-and-error in Wu et al. (2018a), a fresh crater with a diameter of $3-5 \mathrm{M}^{3}$ pixels is optimal to not only distinguish walls and shadows, but also to represent the top unit. Following Wu et al. (2018a) in this study we used the fresh craters with diameters of $400-500 \mathrm{~m}$ to sample spectra and they were from high-quality one pixel to reduce the pixel mixing. In order to ensure that the craters are fresh the very high spatial-resolution LROC Narrow Angle Camera (NAC) data were used. 


\subsection{Spectral parameter acquisition}

The mafic minerals (e.g., pyroxene and olivine) of mare basalts can be identified through their characteristic spectral absorption features. Pyroxene displays two absorption peaks at approximately $1000 \mathrm{~nm}$ (Band I) and $2000 \mathrm{~nm}$ (Band II) (Adams 1974; 1975; Burns 1993; Cloutis 1985; Klima et al. 2007). In contrast, the olivine reflectance spectrum is revealed by a broad and asymmetric $1000 \mathrm{~nm}$ absorption, but lacks the $2000 \mathrm{~nm}$ absorption (Adams 1975; Singer 1981; Burns 1993; Isaacson et al. 2014). The broad Band I absorption in olivine is caused by three distinct absorption bands (Burns 1993). The central absorption, located just beyond $1000 \mathrm{~nm}$, is caused by iron in the M2 crystallographic site. The two weaker absorptions near 850 and $1250 \mathrm{~nm}$ are the result of iron in the M1 site (Burns 1993; Sunshine $\&$ Pieters 1998). The Band I "secondary" absorption near $1250 \mathrm{~nm}$ allows olivine to be detected when admixed with the spectrally "stronger" pyroxene.

The absorption band center is crucial for mineral identification. The Band I and Band II centers provide a qualitative way to access the composition of pyroxenes (Klima et al. 2007). The band centers are influenced by the amount of $\mathrm{Fe}^{2+}$ and $\mathrm{Ca}^{2+}$. For example, with increasing $\mathrm{Fe}^{2+}$ and $\mathrm{Ca}^{2+}$, the band centers move slightly to the longer wavelength (Burns 1993; Hazen et al. 1978). However, in the case of olivine-pyroxene mixtures, Band I is dependent on the relative abundances of both olivine and pyroxene, which makes the interpretations problematic. The band area ratio (BAR), defined as the ratio between the Band II and Band I absorption features, is useful for estimating the relative abundances of pyroxene and olivine in basalts (Cloutis et al. 1986; Gaffey et al. 1993). The BAR is in inversely proportion to the olivine content, but increases linearly with pyroxene abundance (Gaffey et al. 1993; Cloutis et al. 1986).

Prior to calculating the absorption parameters, a B-spline function fitting is used to smoothen the spectral data to reduce the effects of spectral noise. The spectral curve after smoothing maintains a similar form and value to the original, with the noise removed. To derive the accurate absorption band center, the spectral continuum should be removed. The continuum removal was performed using the convex hull method with the $2497 \mathrm{~nm}$ as the right endpoint suggested in $\mathrm{Wu}$ et al. (2018a) to handle the reflectance increases in the longer wavelength. The band area refers to the accumulative value of the absorption depths of the continuum function within 1000 and $2000 \mathrm{~nm}$ band absorption characteristic peaks. They are represented by Band I Area and Band II Area, respectively.

\section{RESULTS AND DISCUSSION}

\subsection{Stratigraphy}

According to the unit division method mentioned above, this study area has been divided into 27 unites, 25 units in Mare Frigoris and Sinus Roris and two units in the northern Mare Imbrium. Kramer et al. (2015) excludes F24 from Mare Frigoris; however, in the LROC WAC and $\mathrm{M}^{3}$ OP1B images, it is smooth and dark on the surface, and thus it is included in the scope of research. Owing to the serious loss of $\mathrm{M}^{3}$ data around Lacus Mortis, it is impossible to extract the spectrum. Thus, it is not included in this study. Fig. 1 shows the unit division results in the $\mathrm{M}^{3}$ IBD image, Clementine color ratio composite image, IIM FeO and $\mathrm{TiO}_{2}$ content diagram, and IIM false color composite image, respectively.

Fig. 1a shows the $\mathrm{M}^{3}$ (IBD) composite. In this figure, the highlands appear blue, whereas mare basalts appear yellow/green to orange according to the relative strength of the mafic minerals. The darker red hues of the unit is, the higher olivine/pyroxene ratios (Staid et al. 2011). In Fig. 1a, the F1, F4, and F6 units are in yellow, and F2, F3, and F5 are in orange. F7, F8, F9, and F10 are in yellowgreen. F11 and F12 are in orange-red. F13 and F15 are in orange-yellow, and F14, F16, F17, and F19 are in yellow-green. Three edge units, namely, F18 in the southern Plato crater and F24 and F25 in the eastern Plato crater, are in purple in the Clementine ratio color composite image (Fig. 1b), and, F18 is in red-purple and F24 is in blue-purple. The blue area in the Clementine ratio false color composite image indicates the high content of titanium in the unit. 


\subsection{Spectral Analysis}

Fig. 2 shows the average reflectance of fresh craters, as well as their average reflectance curves after continuum removal. They are divided into three groups according to the shape of the spectral curves
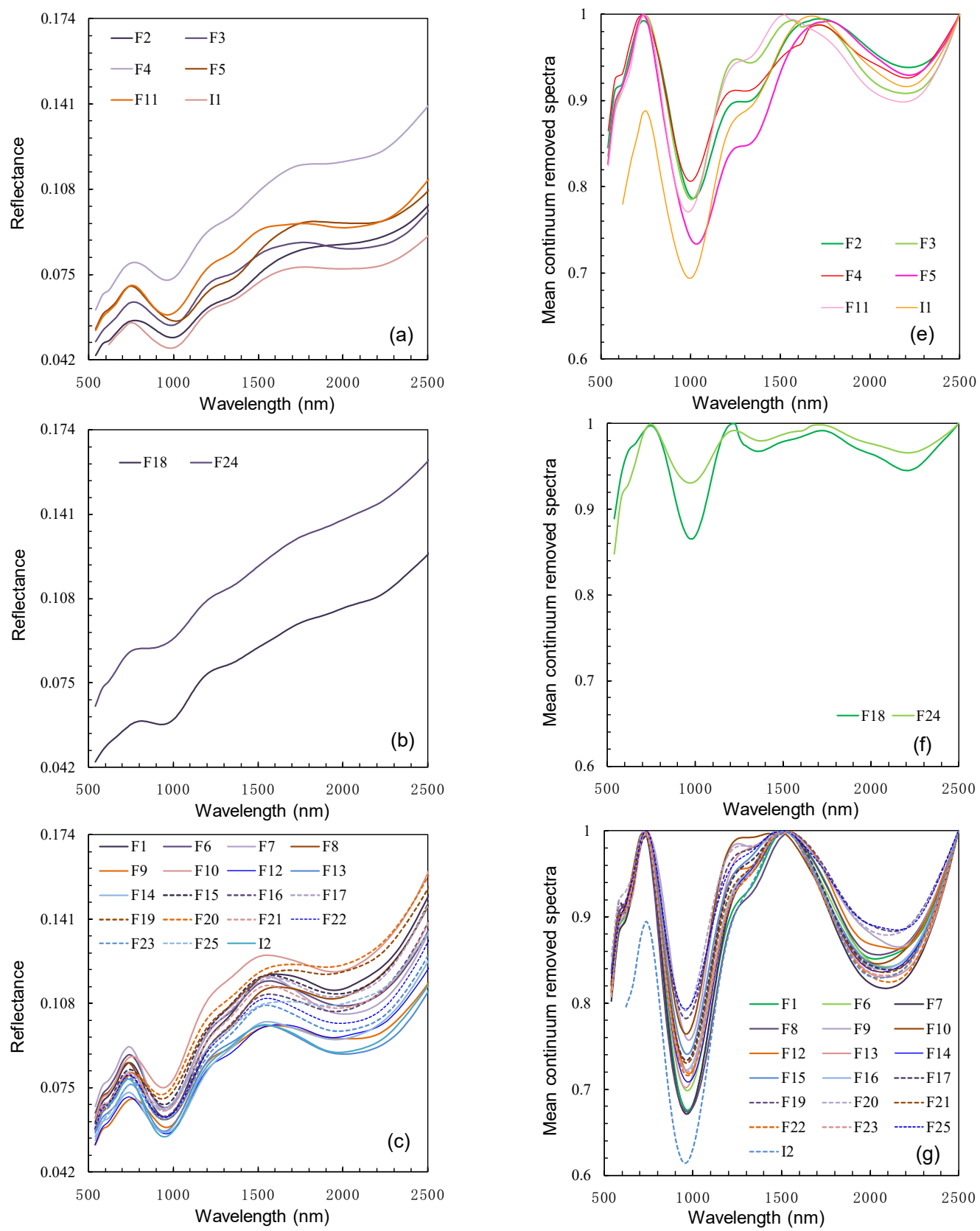

Fig. 2 The average reflectance curves of the different group units and continuum removed spectra of them. (a) G1: Strong absorption at $1000 \mathrm{~nm}$ and weak absorption at $2000 \mathrm{~nm}$; (b) G2: Weak absorption at both $1000 \mathrm{~nm}$ and $2000 \mathrm{~nm}$; (c) G3: Strong absorption at both $1000 \mathrm{~nm}$ and $2000 \mathrm{~nm}$. The continuum removed spectra for (a), (b) and (c) are (e), (f) and (g) respectively. 
(width and depth of absorption characteristic and spectral slope): G1 (F2, F3, F4, and F5 in the western Mare Frigoris; F11 in the central Mare Frigoris; and I1 in the central northern Mare Imbrium), G2 (F18 in the southern Mare Frigoris, and F24 on the extreme eastern side of Mare Frigoris), and G3 (remaining units except those in G1 and G2). The spectra of most units have strong absorption characteristics at 1000 and $2000 \mathrm{~nm}$. The absorption characteristic of five units in G1 at 1000 and $2000 \mathrm{~nm}$ are in the middle level of the three groups. They have an absorption at $1300 \mathrm{~nm}$ and weak absorption at $2000 \mathrm{~nm}$. The absorption characteristic of two units in Group 2 is special; they have the weakest absorption at 1000 and $2000 \mathrm{~nm}$ and a big slope. This may be because it is difficult to select a fresh crater in this region hence contains the mixture of soils, or because the region has less crystallized composition. F24 is the oldest unit in Mare Frigoris, aged 3. $77 \mathrm{Ga}$ (Hiesinger et al. 2010). It has low of iron and titanium contents and a weak absorption characteristic of the spectral curve. The spectral curves of the units in G3 are highly consistent; they have the strongest absorption at 1000 and $2000 \mathrm{~nm}$ and weak absorption at $1300 \mathrm{~nm}$.
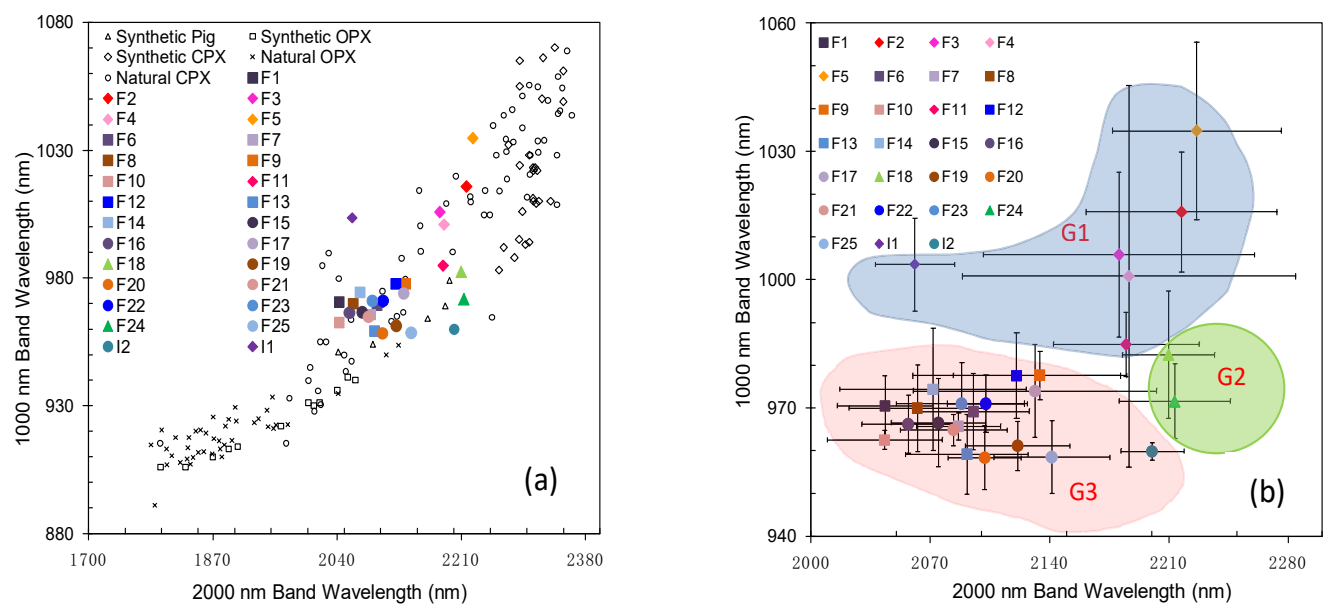

Fig. 3 Plot of the $1000 \mathrm{~nm}$ (Band I) versus $2000 \mathrm{~nm}$ (Band II) absorption center. (a) Mean band center of each unit. The band centers of natural pyroxenes (Adams 1974; Cloutis \& Gaffey 1991) and synthetic pyroxene (Klima et al. 2011) are also included. (b) Mean band center with error bar of each measurement. The error bar represents the standard deviation of each measurement. The blue, green and pink lines circle G1, G2 and G3 group.

Fig. 3 shows the average $1000 \mathrm{~nm}$ and $2000 \mathrm{~nm}$ absorption center scatter plots of all units. As a contrast, the figure also shows the laboratory spectral data of natural pyroxene and synthetic pyroxene (Adams 1974; Klima et al. 2011). The wavelengths of the 1000 and $2000 \mathrm{~nm}$ absorption centers of synthetic low-Ca pyroxene, which better addresses the fundamental constraints of crystal structure and Ca-Mg-Fe content on reflectance spectra, are 900 to $930 \mathrm{~nm}$ and $1800 \mathrm{~nm}$ to $2100 \mathrm{~nm}$, respectively. Those of synthetic high-Ca pyroxene are $950 \mathrm{~nm}$ to $1070 \mathrm{~nm}$ and $2260 \mathrm{~nm}$ to $2400 \mathrm{~nm}$, respectively. G1 has the maximum absorption center wavelength, namely $985 \mathrm{~nm}$ to $1035 \mathrm{~nm}$ and $2181 \mathrm{~nm}$ to 2226 $\mathrm{nm}$, respectively. The spectral curve has a wide and asymmetrical absorption peak at $1000 \mathrm{~nm}$. The absorption peak is weak at $2000 \mathrm{~nm}$, which is the olivine-dominated mineralogy. The absorption center wavelengths of G2 are $972 \mathrm{~nm}$ to $982 \mathrm{~nm}$ and $2210 \mathrm{~nm}$ to $2214 \mathrm{~nm}$, respectively. Those of G3 are 958 $\mathrm{nm}$ to $978 \mathrm{~nm}$ and $2043 \mathrm{~nm}$ to $2141 \mathrm{~nm}$, respectively, indicating rich monoclinic pyroxene; however, the content of pyroxene calcium is lower than that in the lunar samples.

Fig. 3 shows the scatter plot of the absorption center wavelengths and BARs of the units of Mare Frigoris and northern Mare Imbrium. The BAR value is low in G1, ranging from 0.26 to 0.73 , which is the olivine-dominated mineralogy. The BAR value is between 0.76 and 1.52 in G3, which is the clinopy- 
roxenes and olivine mixed mineral. The BARs of F18 and F24 in G2 are 0.85 and 0.83 , respectively.

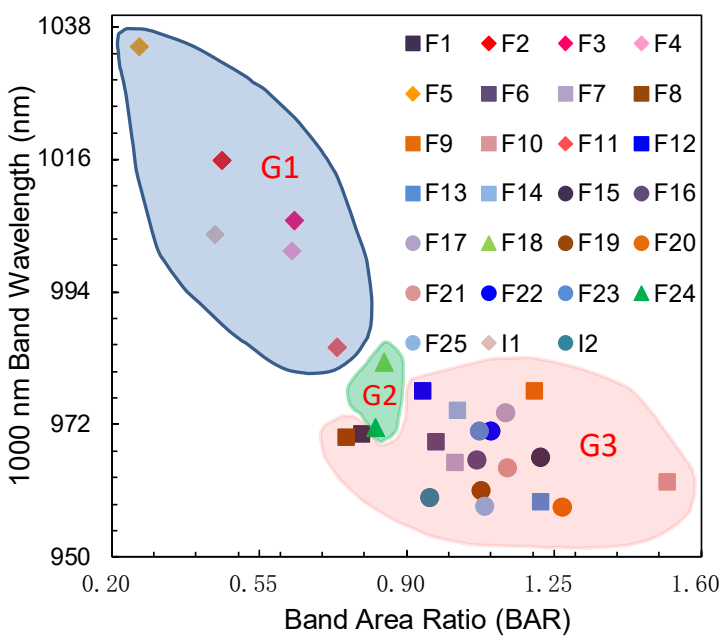

Fig. 4 BAR versus Band I center plot. The blue, green and pink lines circle, G1, G2 and G3, the three groups of different units respectively.

Fig. 4 presents the distribution diagram of the $1000 \mathrm{~nm}$ absorption centers and BAR values of Mare Frigoris and northern Mare Imbrium. Overall, they both show a certain negative correlation, which is in line with the proportional change in olivine/pyroxene. This indicates that the minerals comply with the change from olivine domination to pyroxene domination. The olivine-dominated mineralogy in western Mare Frigoris and central Mare Imbrium has a long $1000 \mathrm{~nm}$ absorption center wavelength and low BAR value. The clinopyroxenes-dominated mineralogy in eastern central Mare Frigoris has the shortest $1000 \mathrm{~nm}$ wavelength and a high BAR value.

The change in the relative trends of mafic mineral in space in Mare Frigoris and northern Mare Imbrium can be seen intuitively from Fig. 5. Western Mare Frigoris, Sinus Roris and central Mare Imbrium (F2, F3, F4, F5, and I1) have the longest $1000 \mathrm{~nm}$ absorption center wavelengths and the smallest BAR, dominated by olivine. Young basalts in Oceanus Procellarum are also dominated by olivine (Staid et al. 2011; Zhang et al. 2016). Further combining with the age data, the basalts in western Mare Frigoris, Sinus Roris, Procellarum, and Mare Imbrium are all found to be young basalts (F2, F3, F4, and F5 aged 1.3-1.7 Ga (Hiesinger et al. 2010), and I1 aged 2.35 Ga (Wu et al. 2018a)). The similar olivine domination and age suggest that the late-stage thermal evolution of the Moon has a regularity in a larger scope.

The Mare Frigoris mineralogy in the northern Plato crater shows different absorption characteristic on the east and west sides. The $1000 \mathrm{~nm}$ absorption center on the west side is long with a small BAR value, whereas that on the east side is short with a large BAR value. The ratio of olivine to pyroxene on the west side is higher than that on the east. The $1000 \mathrm{~nm}$ absorption center wavelength and BAR value of the mineralogy around the eastern Aristoteles crater are $958.3 \mathrm{~nm}$ to $962.5 \mathrm{~nm}$ and 1.02 to 1.14 , respectively. The ratio of olivine to pyroxene is low. The mineralogy in this area is old, aged over $3.56 \mathrm{Ga}$ (Hiesinger et al. 2010). The basalts around the two craters are rich in low-Ca and medium-Ca pyroxene, which is consistent with the Mare Imbrium basalts on the north of the Mare Imbrium basin (Wu et al. 2018a). This may indicate that the deep source of east central Mare Frigoris basalt has certain correlation with those of northern Mare Imbrium basalts. These phenomena indicate that the deep source of Mare Frigoris may be consistent with surrounding adjacent lunar maria, despite being a geographical mare. 


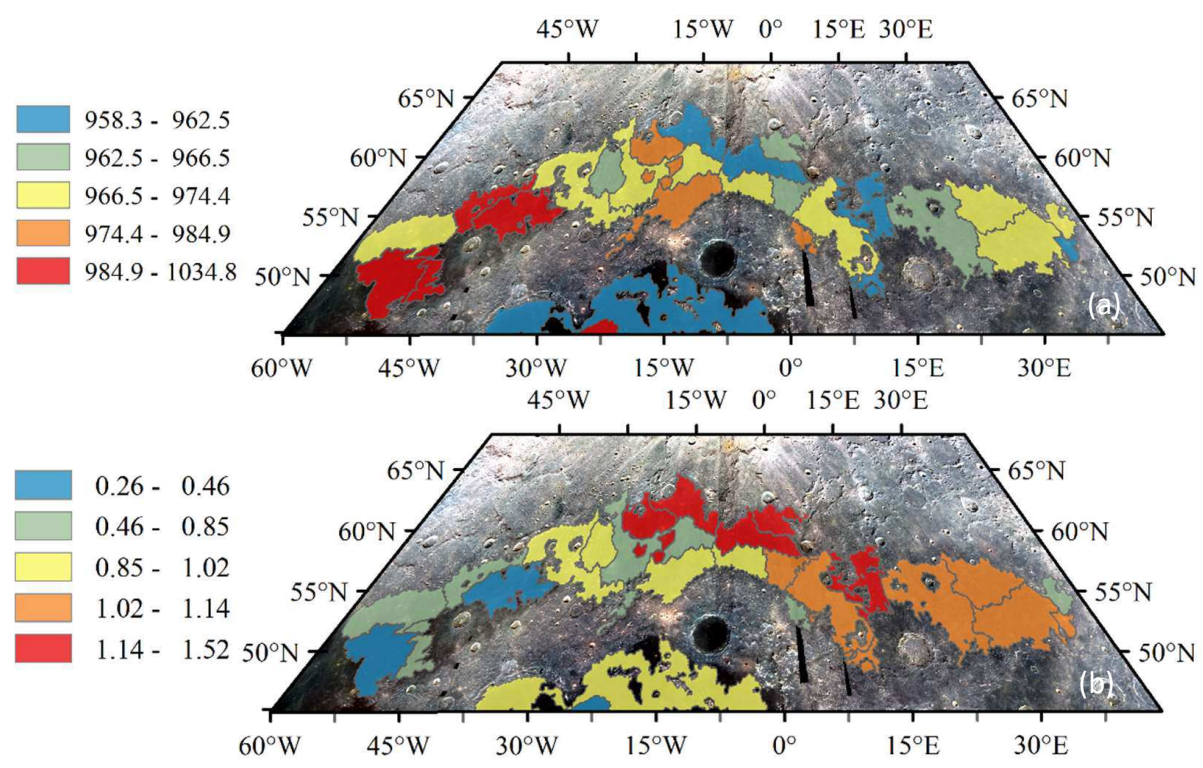

Fig. 5 Distribution of Band I center (a) and BAR (b) values. The base map is the Clementine false color composite image (R-950 nm; G-750 nm; B-415 nm).

\section{SUMMARY AND CONCLUSIONS}

By comprehensive utilization of multi-source data and according to various characteristics of albedo, hue, and topography, this study divided the units of the maria on the northern nearside of the Moon in detail, analyzed the spectral absorption characteristics of different units, mapped the distribution of the $1000 \mathrm{~nm}$ absorption center wavelengths and BAR values, and studied the mineral variation in different units.

The basalts in western Mare Frigoris and Sinus Roris have the highest ratio of olivine to pyroxene, i.e., they are rich in olivine. Similarly, the Eratosthenian basalts in northern Mare Imbrium (and Oceanus Procellarum) are also dominated by olivine (Staid et al. 2011; Varatharajan et al. 2014; Zhang et al. 2016). The basalts in central and eastern Mare Frigoris have low ratios of olivine to pyroxene. The mineralogy with the lowest ratio is located around crater Fontenelle on the southern Mare Frigoris, and is rich in low-Ca to medium-Ca pyroxene. The old basalts in northern Mare Imbrium have similar composition. All of these phenomena indicate that, although Mare Frigoris is a geographical mare, the basalts of Mare Frigoris have different magma sources and have a certain correlation to the sources of adjacent maria.

The unsampled late-stage basalts are spectrally unique hence they are compositionally unique and distinguished from older basalts. For the formation of these spectrally unique basalts some hypotheses have been suggested. One explanation is that the olivine-rich Eratosthenian basalts were formed via the partial melting from the ilmenite-rich mantle source and mixed with the residual KREEP layer beneath the anorthosite crust as it ascended to the surface (Zhang et al. 2015). Alternatively, Zhang et al. (2015) also suggests that the basalts were derived via the partial melting of the mantle source that mixed with sinking ilmenite-rich KREEPy rocks. The young basalts in Mare Frigoris and Sinus Roris are rich in olivine, and the iron and titanium contents are also very high. The composition is similar to that of a large area of young basalts in Oceanus Procellarum and Mare Imbrium, which indicates that the late-stage volcanism of the Moon occurred on a larger scale. The young basalts have the lowest Mg\# among all the rocks of the Moon (e.g., Wu 2012; Crites \& Lucey 2015). The Chang'E-3 rover Active Particle-induced X-ray Spectrometer (APXS) data show that the young basalts are characterized with high-FeO (22.24 wt.\%), medium $\mathrm{TiO}_{2}$ (4.31 wt.\%) and high $\mathrm{Al}_{2} \mathrm{O}_{3}$ (12.11wt.\%) (Wu et al. 2018b). The 
chemical and mineral data indicate that the young basalts do not have a KREEPy composition or affinity (Neal et al. 2015). Therefore, such a large-scale evolution and the unique composition show that their sources have experienced a high evolution process rather than assimilation with KREEPy materials. In the future, the samples of young basalts need to be collected for detailed petrological analysis. Chang'E5 will collect samples, and it is recommended that the late-stage basalts be sampled.

Acknowledgements This research was supported by the National Key R\&D Program of China (2018YFB0504704), the National Natural Science Foundation of China (11773087), the Macau Science and Technology Development Fund (103/2017/A and 119/2017/A3) and Minor Planet Foundation of Purple Mountain Observatory. The $\mathrm{M}^{3}$ Level 2 reflectance data are downloaded from the PDS (http: //pdsimaging.jpl.nasa.gov/volumes/m3.html). The LROC WAC images are acquired from the LROC website (http: //wms.lroc.asu.edu/lroc). The Clementine UV/VIS images are downloaded from the PDS note (http: //pdsimage.wr.usgs.gov).

\section{References}

Adams, J. B. 1974, J. Geophys. Res., 79, 4829

Adams, J. B. 1975, Interpretation of Visible and Near-infrared Diffuse Reflectance Spectra of Pyroxenes and Other Rockforming Minerals, Infrared and Raman Spectroscopy of Lunar and Terrestrial Minerals (Academic Press New York), 91

Burns, R. G. 1993, Mineralogical Applications of Crystal Field Theory, ed. Roger G. Burns (Cambridge, UK: Cambridge Univ. Press), 575

Cloutis, E. A. 1985, Interpretive techniques for reflectance spectra of mafic silicates, M.S. Thesis, University of Hawai' $i$, Honolulu, Hawai' $i$

Cloutis, E. A., Gaffey, M. J., Jackowski, T. L., \& Reed, K. L. 1986, J. Geophys. Res., 91, 11641

Cloutis, E. A., \& Gaffey, M. J. 1991, J. Geophys. Res., 96, 22809

Crites, S. T., \& Lucey, P. G. 2015, Am. Mineral., 100, 973

Gaffey, M. J., Bell, J. F., Brown, R. H., et al. 1993, Icarus, 106, 573

Hazen, R. M., Bell, P. M., \& Mao, H. K. 1978, Lunar and Planetary Science Conference Proceedings, 9, 2919

Head, J. W. 1976, Rev. Geophys., 14, 265

Hiesinger, H., Head, J. W., Wolf, U. et al. 2010, Journal of Geophysical Research (Planets), 115, E03003

Isaacson, P. J., Klima, R. L., Sunshine, J. M. et al. 2014, Am. Mineral., 99, 467

Jolliff, B. L., Gillis, J. J., Haskin, L. A. et al. 2000, J. Geophys. Res., 105, 4197

Kaur, P., Bhattacharya, S., Chauhan, P. et al. 2013, Icarus, 222, 137

Klima, R. L., Pieters C. M., \& Dyar M. D. 2007, Meteorit. Planet. Sci., 42, 235

Klima, R. L., Pieters, C. M., Boardman, J. W., et al. 2011, Journal of Geophysical Research (Planets), 116, E00G06

Kramer, G. Y., Jolliff B. L., \& Neal C. R. 2008, Journal of Geophysical Research (Planets), 113, E01002

Kramer, G. Y., Jaiswal, B., Hawke, B. R. et al. 2015, J. Geophys. Res., 120, 1646

Melosh, H. J. 1989, Impact cratering: A geologic process. Research supported by NASA. New York, Oxford University Press (Oxford Monographs on Geology and Geophysics, No. 11), 253, 11

Neal, C. R., Wu, Y. Z., Cui, X. Z., Peng, W. X., \& Ping, J. S. 2015, Lunar and Planetary Science Conference, 46, 1641

Singer, R. B. 1981, J. Geophys. Res., 86, 7967

Staid, M. I., \& Pieters, C.M. 2001, J. Geophys. Res., 106, 27887

Staid, M. I., Pieters, C. M., Besse, S., et al. 2011, Journal of Geophysical Research (Planets), 116, E00G10

Sunshine, J. M., \& Pieters C. M. 1998, J. Geophys. Res., 103, 13675

Varatharajan, I., Srivastava, N., \& Murty, S. V. S. 2014, Icarus, 236, 56 
Whitford-Stark, J. L. 1990, Proc. Lunar Sci. Conf., 20th, 175

Whitten, J., Head, J. W., Staid, M. et al. 2011, Journal of Geophysical Research (Planets)., 116, E00G09

Wu, Y. Z. 2012, Geochim. Cosmochim. Acta, 93, 214

Wu, Y. Z., Besse, S., Li, J. Y. et al. 2013, Icarus, 222, 283

Wu, Y. Z., Li, L., Luo, X. et al. 2018a, Icarus, 303, 67

Wu, Y., Wang, Z., Cai, W., \& Lu, Y. 2018b, Astron. J., 155, 213

Zhang, J., Yang, W., Hu, S. et al. 2015, Proc. Natl. Acad. Sci., 112, 5342

Zhang, X., Wu, Y., Ouyang, Z. et al. 2016, J. Geophys. Res., 121, 2063 